\title{
Penumbral models in the light of Hinode spectropolarimetric observations
}

\author{
J. Jurčák ${ }^{1,2}$ and L. R. Bellot Rubio ${ }^{1,3}$ \\ 1 National Astronomical Observatory of Japan, 2-21-1 Osawa, Mitaka, Tokyo 181-8588, Japan \\ e-mail: jan.jurcak@nao.ac.jp \\ 2 Astronomical Institute of the Academy of Sciences, Fricova 298, 25165 Ondřejov, Czech Republic \\ 3 Instituto de Astrofísica de Andalucía (CSIC), Apdo. Correos 3004, 18080 Granada, Spain
}

Received 8 November 2007 / Accepted 16 January 2008

\section{ABSTRACT}

\begin{abstract}
Aims. The realism of current models of the penumbra is assessed by comparing their predictions with the plasma properties of penumbral filaments as retrieved from spectropolarimetric observations.

Methods. The spectropolarimeter onboard Hinode allows us, for the first time, to distinguish the fine structure of the penumbra. Therefore, we can use one-component inversions to obtain the stratifications of plasma parameters in each pixel. The correlations between the plasma parameters and the continuum intensity are studied.

Results. We find that, in the outer penumbra, the stronger flows and higher values of magnetic field inclination tend to be located in dark filaments. This finding does not seem to be compatible with the scenario of a field-free gappy penumbra.
\end{abstract}

Key words. Sun: sunspots - Sun: photosphere - techniques: polarimetric

\section{Introduction}

Currently, there are two models of the penumbral fine structure under discussion. The first is the uncombed model proposed by Solanki \& Montavon (1993) and further developed by Martínez Pillet (2000) to explain the broadband circular polarisation observed in sunspots. It envisages the penumbra as a collection of horizontal flux tubes embedded in a more vertical and stronger background field. Schlichenmaier et al. (1998) made simulations of the temporal evolution of such flux tubes. Initially placed at the sunspot magnetopause, they rise by magnetic buoyancy and quickly become horizontal in the photosphere. At the same time, a strong Evershed flow develops due to gas pressure gradients that build up during the rise. The tubes are hotter than the surrounding plasma and appear as bright penumbral filaments. These filamentsgradually cool down due to radiative losses, i.e. they become dark further from the bright penumbral grains where the tubes cross the $\tau=1$ layer. Hereafter, this model will be called the rising flux tube model (RFT).

The second model was suggested by Spruit \& Scharmer (2006). These authors argued that the RFT model cannot produce sufficient heating of the penumbra. As an alternative, they proposed a field-free gap model (FFG) which consists of fieldfree material protruding into the penumbral magnetic field from below. With such a configuration, the bright filaments would be heated all along their lengths and not only at the point of emergence as in the case of the RFT model. While the FFG model does not offer any explanation for the Evershed flow, the flow can only reside on top of the field-free gaps since this is the sole region in the model where horizontal fields exist.

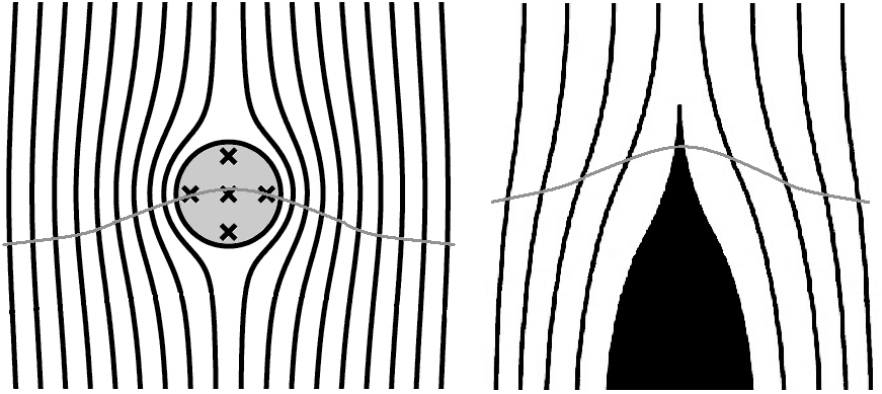

Fig. 1. Sketch of a RFT (left) and a FFG (right) surrounded by the background magnetic field of the penumbra. The gray line represents the possible position of the optical depth unity layer.

Both models have problems from a theoretical point of view that need to be clarified (see Bellot Rubio 2007, and references therein). However, as illustrated in Fig. 1, RFTs and FFGs should produce similar stratifications of the plasma parameters if they are placed near $\tau=1$ (marked by the gray line). The two models predict weaker and more inclined fields low in the atmosphere, along with increased line-of-sight (LOS) velocities. Using Hinode spectropolarimetric data, Jurčák et al. (2007) found this configuration in the bright filaments of the inner penumbra.

In this Letter, we concentrate on the middle and outer penumbra, where differences between the models could be more pronounced. According to the FFG model, horizontal fields and increased LOS velocities should be associated with bright areas everywhere in the penumbra. By contrast, the RFT model 


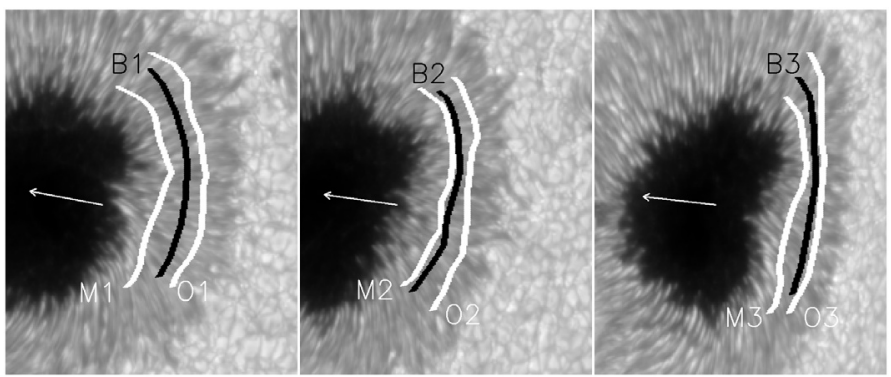

Fig. 2. Maps of continuum intensity at $630.3 \mathrm{~nm}$ reconstructed from the $\mathrm{SP}$ raster scans. North is up and West to the right. The maps cover the limbside part of the penumbra in evolving AR 10930. The arrows point to the disc centre. The black and white bands indicate the areas selected for analysis.

expects such characteristics in the dark filaments of the outer penumbra.

\section{Observations and data analysis}

The data analysed here were obtained using the spectropolarimeter (SP; Tarbell et al. 2007) of the Solar Optical Telescope (Tsuneta et al. 2007) onboard the Hinode satellite (Kosugi et al. 2007). This instrument measures the Stokes profiles of the two iron lines at $630.15 \mathrm{~nm}$ and $630.25 \mathrm{~nm}$.

Normal SP scans of AR 10930, providing a spatial resolution of 0.32 and a noise level of $10^{-3} I_{c}$, were taken on December 13,14 , and 15, 2006, when the spot was located at heliocentric angles of $30^{\circ}, 41^{\circ}$, and $54^{\circ}$, respectively. The three panels of Fig. 2 show the limbside penumbra of AR 10930 as reconstructed from the continuum intensities observed redward of the $630.25 \mathrm{~nm}$ line. The Stokes $V$ profiles emerging from the penumbra exhibit significant area asymmetries due to the existence of gradients of the plasma parameters in the line-forming region. We can clearly see the bright and dark filaments in the continuum maps. Their signals are not mixed as in the case of groundbased observations, implying that the penumbral fine structure is distinguished in spectropolarimetric measurements for the first time. Higher spatial resolution observations of the penumbra exist (e.g., Scharmer et al. 2002; Rouppe van der Voort et al. 2004; Rimmele 2004; Langhans et al. 2005; Bellot Rubio et al. 2005; Sánchez Almeida et al. 2007), but they correspond to filtergrams, Dopplergrams, magnetograms, and spectroscopic measurements from which the magnetic field vector cannot be determined.

Each day we selected three 5-pixel-wide azimuthal cuts. As illustrated in Fig. 2, they sample the middle and outer parts of the penumbra. The bands were chosen so as to minimise any trend of the continuum intensity along them, except for small-scale fluctuations caused by the alternating bright and dark filaments. In total, they contain 5862 pixels.

The data reduction is described in detail in Jurčák et al. (2007). The zero point of the velocity scale is taken to be the line core position of the mean quiet Sun intensity profile, computed separately for each slit. The observed Stokes spectra have been inverted using the SIRGAUS code (Bellot Rubio 2003), which is a modified version of SIR (Stokes Inversion based on Response function; Ruiz Cobo \& del Toro Iniesta 1992). This code presumes the existence of a Gaussian perturbation (GP) in the stratifications of plasma parameters somewhere in the lineforming region. Given the high angular resolution of the Hinode measurements, we only consider one-component model atmospheres.

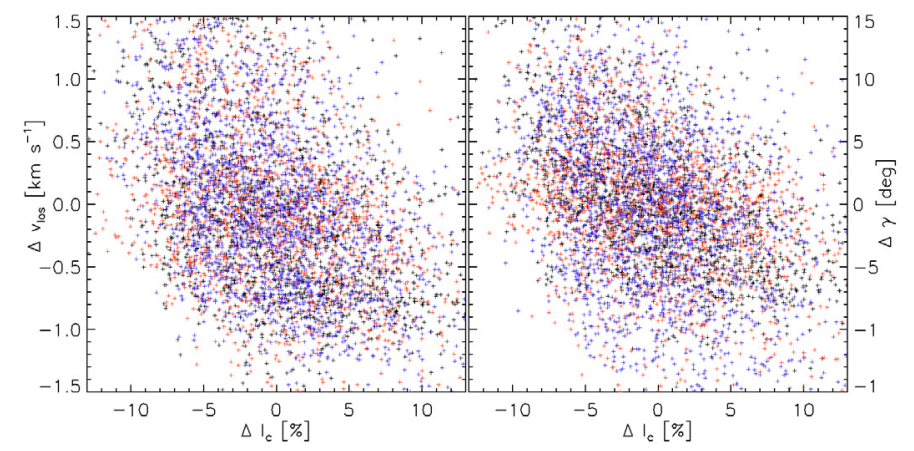

Fig. 3. Scatter plots of LOS velocity (left) and inclination (right) fluctuations vs. continuum intensity fluctuations. The black, red, and blue symbols represent points from the $\mathrm{M}, \mathrm{B}$, and $\mathrm{O}$ bands, respectively.

The inversion code looks for the best solution in a space of 13 free parameters. Six of those parameters define the physical conditions of the unperturbed atmosphere: we use two for temperature $(T)$, and one for field strength $(B)$, inclination $\left(\gamma_{\mathrm{LOS}}\right)$, azimuth $\left(\phi_{\mathrm{LOS}}\right)$, and LOS velocity $\left(v_{\mathrm{LOS}}\right)$. The width and position of the GP are the same for all plasma parameters, which adds two more free parameters. The rest of the parameters are the amplitudes of the perturbation in the different physical quantities. As initial values, we adopt $\Delta T=+800 \mathrm{~K} ; \Delta B=-500 \mathrm{G}$; $\Delta \gamma_{\mathrm{LOS}}=-30^{\circ} ; \Delta \phi_{\mathrm{LOS}}=-5^{\circ} ;$ and $\Delta v_{\mathrm{LOS}}=+3 \mathrm{~km} \mathrm{~s}^{-1}$. These initial parameters imply weaker and more inclined fields associated with stronger Evershed flows, so they can model both RFTs and FFGs. The magnetic field inclination and azimuth retrieved by the inversion code are line-of-sight values. We transformed these variables to the local reference frame. In what follows, the field inclination $(\gamma)$ represents the angle between the magnetic field vector and the inward normal to the solar surface.

\section{Choice of plasma parameters}

As explained by Jurčák et al. (2007), we perform four inversions per pixel with different starting heights of the GP, and select the one that shows the smallest value of the merit function (the sum of squared differences between observed and synthetic profiles). Sometimes, the Stokes spectra emerging from a given pixel can be fitted equally well by GPs located either in deep layers or in the middle photosphere. In theose cases, it is difficult to decide which solution is closer to reality on the basis of the merit functions alone, as they are usually of comparable value. Although the resulting stratifications are different, they share some similarities: variations in the position of the GP mainly change the amplitude and sign of the magnetic field strength perturbation, but the sign and amplitude of the GP for the LOS velocity and the field inclination are almost independent of the height of the Gaussian.

We take advantage of this fact by adopting the part of the solution that is similar in all cases, i.e., the average values of the plasma parameters in the line-forming region. Specifically, for each pixel we compute the mean value of the LOS velocity, field inclination, and field strength in the range from $\log \tau=-0.5$ to $\log \tau=-2.5$. This is the region where the Stokes profiles of the Fe I $630.2 \mathrm{~nm}$ lines are more sensitive to the physical conditions of the atmosphere (Cabrera Solana et al. 2005).

From the obtained values, we subtract the locally averaged plasma parameters, as defined by a running mean over 100 pixels. The resulting fluctuations of continuum intensity, LOS velocity, field inclination, and field strength are used to test the predictions of the RFT and FFG models. 


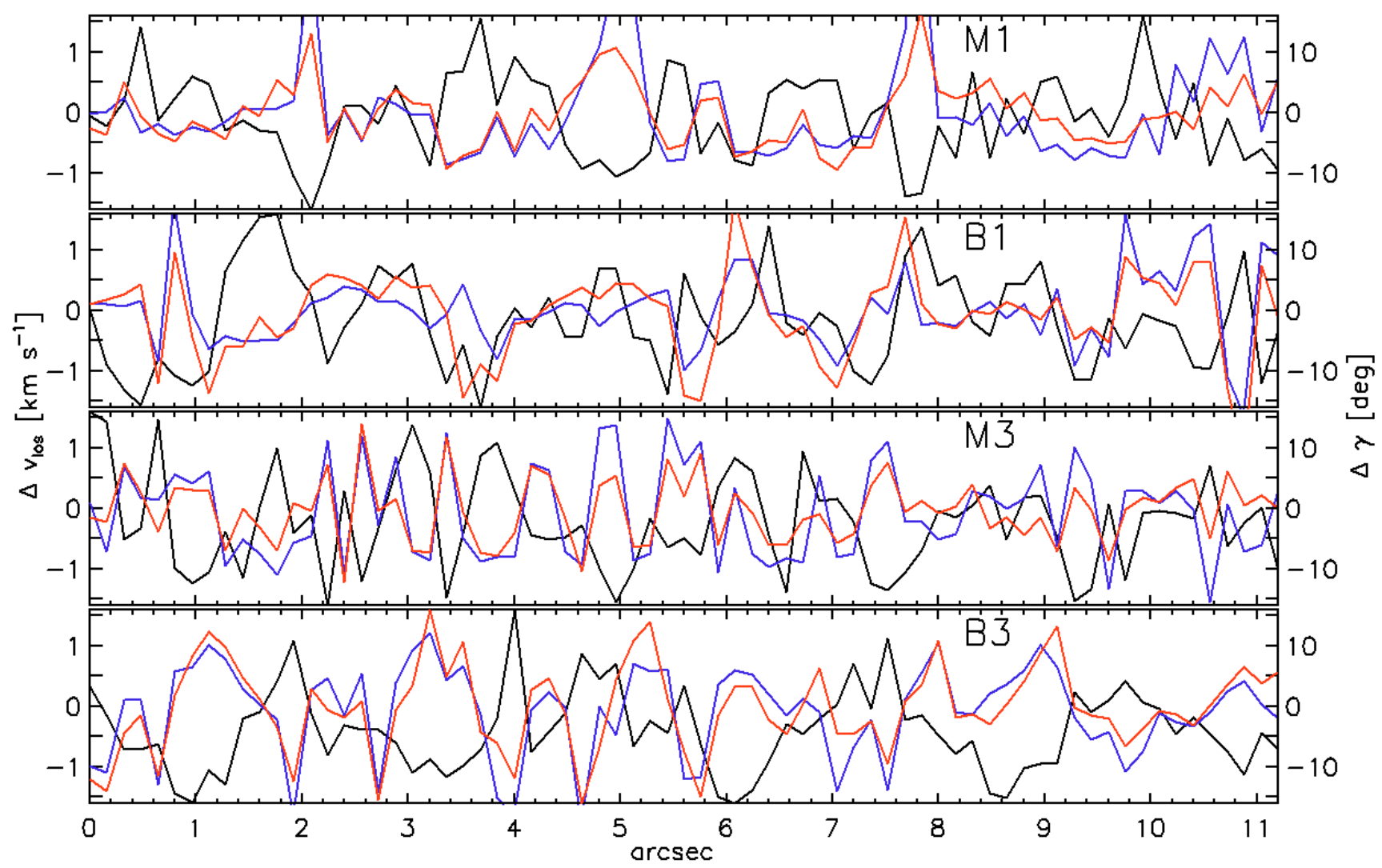

Fig. 4. Continuum intensity (black), LOS velocity (blue), and magnetic field inclination (red) fluctuations along the selected paths. The plots show, from top to bottom, parts of the M1, B1, M3, and B3 bands.

\section{Results}

Figure 3 shows scatter plots of LOS velocity and magnetic field inclination fluctuations vs. continuum intensity fluctuations. There is a clear tendency for weaker flows and more vertical fields in brighter penumbral areas. However, this type of plot does not reflect how the plasma parameters are associated with penumbral filaments. Therefore, in Fig. 4 we give examples of continuum intensity fluctuations (black lines) and the corresponding changes of LOS velocity (blue lines) and inclination (red lines) along one-pixel-wide azimuthal cuts extracted from the selected bands.

Table 1 summarises the correlation coefficients obtained in the nine bands, with errors representing 95\% confidence intervals. The values shown in the second and third columns confirm the visual impression from Figs. 3 and 4 that, in the middle and outer penumbra, the stronger flows and the more inclined fields usually occur in dark filaments. The average correlation coefficient between $\Delta v_{\mathrm{LOS}}$ and $\Delta I_{\mathrm{c}}$ amounts to -0.40 , which is quite high compared to previous analyses based on spectropolarimetric data (e.g., Westendorp Plaza et al. 2001; Solanki 2003). Enhanced LOS velocities in dark filaments were also found from high-resolution filtergrams with comparable or even better correlations (e.g., Schlichenmaier et al. 2005; Ichimoto et al. 2007).

The third column of Table 1 shows the correlation coefficients between $\Delta \gamma$ and $\Delta I_{\mathrm{c}}$. In this case the average correlation is $-0.40(-0.46$ if we do not take into account bands B1 and $\mathrm{O} 1$, which are significantly worse). Thus, there is a tendency for the more horizontal fields to be associated with dark penumbral areas. Previous analyses reported either no significant correlation between these parameters in the outer penumbra (Title et al. 1993; Stanchfield et al. 1997), or larger field inclinations
Table 1. Correlation coefficients between fluctuations of LOS velocity and continuum intensity (second column); field inclination and continuum intensity (third column); field inclination and LOS velocity (fourth column); and field inclination and strength (fifth column); along the nine bands marked in Fig. 2. Errors represent 95\% confidence intervals.

\begin{tabular}{ccccc}
\hline \hline & $\Delta v_{\text {LOS }}$ & $\Delta \gamma$ & $\Delta \gamma$ & $\Delta \gamma$ \\
Cut & vs. $\Delta I_{\text {c }}$ & vs. $\Delta I_{\text {c }}$ & vs. $\Delta v_{\text {LOS }}$ & vs. $\Delta B$ \\
\hline M1 & $-0.56 \pm 0.06$ & $-0.55 \pm 0.06$ & $0.82 \pm 0.03$ & $-0.78 \pm 0.04$ \\
M2 & $-0.23 \pm 0.08$ & $-0.44 \pm 0.07$ & $0.78 \pm 0.03$ & $-0.75 \pm 0.04$ \\
M3 & $-0.44 \pm 0.07$ & $-0.40 \pm 0.07$ & $0.78 \pm 0.03$ & $-0.69 \pm 0.04$ \\
B1 & $-0.34 \pm 0.08$ & $-0.15 \pm 0.09$ & $0.81 \pm 0.03$ & $-0.72 \pm 0.04$ \\
B2 & $-0.36 \pm 0.07$ & $-0.53 \pm 0.05$ & $0.67 \pm 0.04$ & $-0.71 \pm 0.04$ \\
B3 & $-0.41 \pm 0.07$ & $-0.43 \pm 0.06$ & $0.70 \pm 0.04$ & $-0.46 \pm 0.06$ \\
O1 & $-0.30 \pm 0.07$ & $-0.18 \pm 0.07$ & $0.86 \pm 0.02$ & $-0.30 \pm 0.07$ \\
O2 & $-0.42 \pm 0.06$ & $-0.44 \pm 0.06$ & $0.77 \pm 0.04$ & $-0.33 \pm 0.07$ \\
O3 & $-0.55 \pm 0.05$ & $-0.45 \pm 0.06$ & $0.60 \pm 0.04$ & $-0.30 \pm 0.06$ \\
\hline
\end{tabular}

in dark filaments (e.g., Rimmele 1995; Westendorp Plaza et al. 2001; Langhans et al. 2005). Usually, the correlation coefficients quoted in those works are smaller (in absolute value) than 0.2. Only when the correlation is analysed at small spatial scales do the coefficients become similar to (or higher than) the ones reported here.

The two last columns of Table 1 quantify the relation between the changes of magnetic field inclination and the LOS velocity and field strength. It is generally accepted that the Evershed flow resides in areas of horizontal fields. With correlation coefficients as high as $0.7-0.8$, the fourth column of Table 1 just confirms this fact. The fifth shows that the more inclined fields are weaker, although the correlation coefficients decrease 
significantly from the middle to the outer penumbra. This implies a smaller difference in magnetic field strength between areas with horizontal fields (or flow channels) and the surrounding atmosphere in the outer penumbra, which is not surprising as both the RFT and FFG models predict an equalisation of field strength with increasing distance from the umbra. A smaller field strength difference between inclined flow channels and surrounding plasma has also been inferred from two-component Stokes inversions (e.g., Bellot Rubio et al. 2004; Borrero et al. 2006), and analyses of the net circular polarisation observed in the penumbra at high angular resolution (Tritschler et al. 2007).

The plasma parameter averages made over the line-forming region do not tell us much about the actual properties of the flow channels represented by the GP. Therefore, we also studied the general behaviour of the peak values of the GPs of LOS velocity and magnetic field inclination. We found that the flow becomes faster with increasing distance from the umbra (on average it is $2 \mathrm{~km} \mathrm{~s}^{-1}$ faster in the $\mathrm{O}$ cuts than in the $\mathrm{M}$ cuts). This increase confirms previous reports of enhanced flow velocity with increasing distance from the umbra (e.g., Rimmele 1995; Westendorp Plaza et al. 2001; Solanki 2003; Bellot Rubio et al. 2004; Borrero et al. 2006), and is also predicted by simulations of moving penumbral tubes (Schlichenmaier et al. 1998).

The maximum values of the LOS velocity are around $10 \mathrm{~km} \mathrm{~s}^{-1}$ near the symmetry line in the O2, B3, and O3 cuts. Even if such velocities are overestimated because they represent the peak amplitudes of the GP, the absolute flow velocity must be very high due to projection effects. These velocities are compatible with the supersonic flows predicted by the simulations of Schlichenmaier et al. (1998) in the outer penumbra.

The average magnetic field inclinations in the flow channels are $85^{\circ}, 100^{\circ}$, and $105^{\circ}$ in the $\mathrm{M}, \mathrm{B}$, and $\mathrm{O}$ cuts, respectively. This means that the flow channels are inclined upward in the middle penumbra and point down in the outer penumbra (the polarity of the spot is negative). Magnetic fields returning to the solar surface in the outer penumbra were already reported by, e.g., Westendorp Plaza et al. (2001) and Bellot Rubio et al. (2004).

\section{Discussion and conclusions}

The most important result of our analysis is that we do not find positive correlation coefficients between $I_{\mathrm{c}}$ and $\gamma$ or $v_{\mathrm{LOS}}$. This indicates that bright penumbral filaments show the more vertical fields and weaker flows, in contradiction with the predictions of the FFG model. The horizontal fields and stronger flows tend to be associated with dark filaments, as has been reported previously from lower resolution data. The fact that the correlation coefficients between $I_{\mathrm{c}}$ and $\gamma$ or between $I_{\mathrm{c}}$ and $v_{\mathrm{LOS}}$ are not -1 can be easily explained by the mixing of flux tubes and background magnetic fields. In the middle and outer penumbra, dark areas may represent either the darkened tails of RFTs or background fields. The latter are characterised by more vertical fields and weaker flows. Such an opposite behaviour decreases the correlation coefficients, which nevertheless remain negative because of the overall dominance of dark RFTs in the outer parts of the penumbra. This argument does not influence the correlation coefficients between $\gamma$ and $v_{\text {LOS }}$, which are indeed much higher than those involving $I_{\mathrm{c}}$.

Schlichenmaier et al. (2005) and Ichimoto et al. (2007) came to a similar conclusion (regarding the velocities) from simpler interpretations of filtergrams and spectropolarimetric data, respectively. They also reported that the horizontal fields and the flows are associated with bright filaments in the innermost penumbra (see also Hirzberger et al. 2005; Jurčák et al. 2007), where the dark areas most likely represent background magnetic fields. Thus, the correlation coefficients between continuum intensity and LOS velocity/inclination change sign from the inner to the outer penumbra due to the fact that RFTs predominantly show up as bright structures in the innermost penumbra, but darken toward the outer edge of the spot. This sign reversal cannot be explained by the FFG model. The existence of magnetic field lines pointing to the solar interior in the outer penumbra, also confirmed by our observations, represents another problem for the FFG model (Bellot Rubio 2007).

The RFT simulations of Schlichenmaier et al. (1998) seem to be able to explain all the results of our analysis, including the sign reversal of the correlation coefficients and the larger mean value of the LOS velocity in the outer penumbra as compared with the middle penumbra. Overall, this suggests that the RFT model provides a better description of the penumbra than the FFG model.

Acknowledgements. This work has been enabled thanks to the funding provided by the Japan Society for the Promotion of Science. Hinode is a Japanese mission developed and launched by ISAS/JAXA, with NAOJ as domestic partner and NASA and STFC (UK) as international partners. It is operated by these agencies in cooperation with ESA and NSC (Norway). The computations were carried out at the NAOJ Hinode Science Center, which is supported by the Grant-in-Aid for Creative Scientic Research The Basic Study of Space Weather Prediction from MEXT, Japan (Head Investigator: K. Shibata), generous donations from Sun Microsystems, and NAOJ internal funding. Financial support from the Spanish Ministerio de Educación y Ciencia through project ESP2006-13030-C06-02 is gratefully acknowledged.

\section{References}

Bellot Rubio, L. R. 2003, ASP Conf. Ser., 307, 301

Bellot Rubio, L.R. 2007, in Highlights of Spanish Astrophysics IV, ed. F. Figueras et al. (Dordretch: Springer), 271

Bellot Rubio, L. R., Balthasar, H., \& Collados, M. 2004, A\&A, 427, 319

Bellot Rubio, L. R., Langhans, K., \& Schlichenmaier, R. 2005, A\&A, 443, L7

Borrero, J. M., Solanki, S. K., Lagg, A., Socas-Navarro, H., \& Lites, B. 2006, A\&A, 450, 383

Cabrera Solana, D., Bellot Rubio, L.R., \& del Toro Iniesta, J.C. 2005, A\&A, 439,687

Hirzberger, J., Stangl, S., Gersin, K., et al. 2005, A\&A, 442, 1079

Ichimoto, K., Shine, R.A., Lites, B.W., et al. 2007, PASJ, 59, 593

Jurčák, J., Bellot Rubio, L.R., Ichimoto, K., et al. 2007, PASJ, 59, 601

Kosugi, T., Matsuzaki, K., Sakao, T., et al. 2007, Sol. Phys., 243, 3

Langhans, K., Scharmer, G. B., Kiselman, D., Löfdahl, M. G., \& Berger, T. E. 2005, A\&A, 436, 1087

Martínez Pillet, V. 2000, A\&A, 361, 734

Rimmele, T. R. 1995, A\&A, 298, 260

Rimmele, T. R. 2004, ApJ, 604, 906

Rouppe van der Voort, L. H. M., Löfdahl, M. G., Kiselman, D., \& Scharmer, G. B. 2004, A\&A, 414, 717

Ruiz Cobo, B., \& del Toro Iniesta, J. C. 1992, ApJ, 398, 375

Sánchez Almeida, J., Márquez, I., Bonet, J. A., \& Domínguez Cerdeña, I. 2007, ApJ, 658, 1357

Scharmer, G. B., Gudiksen, B. V., Kiselman, D., Löfdahl, M. G., \& Rouppe van der Voort, L. H. M. 2002, Nature, 420, 151

Schlichenmaier, R., Jahn, K., \& Schmidt, H. U. 1998, A\&A, 337, 897

Schlichenmaier, R., Bellot Rubio, L. R., \& Tritschler, A. 2005, AN, 326, 301

Solanki, S. K. 2003, A\&ARv, 11, 153

Solanki, S. K., \& Montavon, C. A. P. 1993, A\&A, 275, 283

Spruit, H. C., \& Scharmer, G. B. 2006, A\&A, 447, 343

Stanchfield, D. C. H., II, Thomas, J. H., \& Lites, B. W. 1997, ApJ, 477, 485

Tarbell, T., et al. 2007, Sol. Phys., in preparation

Title, A. M., Frank, Z. A., Shine, R. A., et al. 1993, ApJ, 403, 780

Tsuneta, S., Ichimoto, K., Katsukawa, Y., et al. 2007, Sol. Phys., submitted

Tritschler, A., Mueller, D. A. N., Schlichenmaier, R., \& Hagenaar, H. J. 2007, ApJ, in press, [arXiv: 0710.4545]

Westendorp Plaza, C., del Toro Iniesta, J. C., Ruiz Cobo, B., \& Martínez Pillet, V. 2001, ApJ, 547, 1148 